\title{
Technical Note: The normal quantile transformation and its application in a flood forecasting system
}

\author{
K. Bogner ${ }^{1}$, F. Pappenberger ${ }^{2}$, and H. L. Cloke ${ }^{2,3}$ \\ ${ }^{1}$ Institute for Environment and Sustainability, European Commission Joint Research Centre, Via E. Fermi, 2749 , \\ 21027 Ispra, VA, Italy \\ ${ }^{2}$ European Centre for Medium-Range Weather Forecasts Shinfield Park Reading, Reading, RG2 9AX, UK \\ ${ }^{3}$ Department of Geography, King's College London, London, UK \\ Correspondence to: K. Bogner (konrad.bogner@jrc.ec.europa.eu)
}

Received: 26 September 2011 - Published in Hydrol. Earth Syst. Sci. Discuss.: 17 October 2011

Revised: 7 February 2012 - Accepted: 22 March 2012 - Published: 2 April 2012

\begin{abstract}
The Normal Quantile Transform (NQT) has been used in many hydrological and meteorological applications in order to make the Cumulated Distribution Function (CDF) of the observed, simulated and forecast river discharge, water level or precipitation data Gaussian. It is also the heart of the meta-Gaussian model for assessing the total predictive uncertainty of the Hydrological Uncertainty Processor (HUP) developed by Krzysztofowicz. In the field of geo-statistics this transformation is better known as the Normal-Score Transform. In this paper some possible problems caused by small sample sizes when applying the NQT in flood forecasting systems will be discussed and a novel way to solve the problem will be outlined by combining extreme value analysis and non-parametric regression methods. The method will be illustrated by examples of hydrological stream-flow forecasts.
\end{abstract}

\section{Introduction}

The Normal Score Transform or NQT has been applied in various fields of geo-science in order to make the mostly asymmetrical distributed real world observed variables more treatable and to fulfil the basic underlying assumption of normality, which is intrinsic to most statistical models (e.g. Moran, 1970; Goovaerts, 1997). For example the metaGaussian model is constructed by embedding the NQT of each variate into the Gaussian law (Kelly and Krzysztofowicz, 1997), which allows the marginal distribution functions of the variates to take any form and the dependence structure between any two variates to be monotone non-linear and heteroscedastic. This most convenient property has been incorporated into the HUP (Krzysztofowicz and Kelly, 2000; Krzysztofowicz and Herr, 2001; Krzysztofowicz and Maranzano, 2004), which is now part of several operational forecasting systems (e.g. Reggiani et al., 2009; Bogner and Pappenberger, 2011) in order to estimate the predictive uncertainty of the hydrological forecasts. In Montanari and Brath (2004) and Montanari and Grossi (2008) some problems of the NQT are discussed regarding its limited possibility of making the probability distribution of bivariate random variables multivariate Gaussian. However, Bogner and Pappenberger (2011) demonstrated that the application of error correction methods could minimize these problems in the case of flood forecasting purposes significantly.

In Van der Waerden $(1952,1953 a, b)$ the theory behind the NQT is outlined and the practical application is demonstrated (e.g. in Krzysztofowicz, 1997; Montanari, 2005; Seo et al., 2006; Todini, 2008). The main objective of this study is to show the difficulties occurring in the inversion of the empirical NQT, if the normal random deviates lie outside the range of the historically observed range, which is particularly important, if this happens during the forecast lead-time.

Several approaches have been been applied in order to solve this critical point of the NQT, for example in Seo et al. (2006) the empirical CDF's are extended beyond the historical maxima by the hyperbolic approximation for the uppermost-tail of the distribution (Deutsch and Journel, 1998). Similar to that approach Coccia and Todini (2011) fitted some additional models for the lower and upper tails 
Table 1. River Gauging station information for Bohumin and Hofkirchen including stream-flow characteristic value MQ (mean discharge) and MHQ (average yearly mean discharge).

\begin{tabular}{llrcrrrc}
\hline Station & River & $\begin{array}{r}\text { Area } \\
\left(\mathrm{km}^{2}\right)\end{array}$ & $\begin{array}{c}\text { Channel gradient } \\
\left(\mathrm{m} \mathrm{m}^{-1}\right)\end{array}$ & $\begin{array}{r}\text { MQ } \\
\left(\mathrm{m}^{3} \mathrm{~s}^{-1}\right)\end{array}$ & $\begin{array}{r}\text { MHQ } \\
\left(\mathrm{m}^{3} \mathrm{~s}^{-1}\right)\end{array}$ & $\begin{array}{c}\text { Latitude } \\
\left({ }^{\circ}\right)\end{array}$ & $\begin{array}{c}\text { Longitude } \\
\left({ }^{\circ}\right)\end{array}$ \\
\hline Bohumin & Oder & 4350 & 0.008 & 40 & 350 & 49.92 & 18.33 \\
Hofkirchen & Danube & 48000 & 0.005 & 640 & 1870 & 48.68 & 13.11 \\
\hline
\end{tabular}

of the variables by setting the maximum value, for which the probability is assumed to be equal to one, to twice the maximum value of the observed historical maximum, resp. to zero for the minimum value, for which the probability is assumed to be null. In Weerts et al. (2011) a linear extrapolation is applied on a number of points in the tails of the distribution. In this study the approach of applying extreme value theory and non-parametric methods will be analysed in more detail.

Following the work of Krzysztofowicz (1997) the empirical NQT involves the following steps:

1. Sorting the sample $X$ from the smallest to the largest observation, $x_{(1)}, \ldots, x_{(n)}$.

2. Estimating the cumulative probabilities $p_{(i)}, \ldots, p_{(n)}$ using a plotting position like $i /(n+1)$ such that $p_{(i)}=P\left(X \leq x_{(i)}\right)$.

3. Transforming each observation $x_{(i)}$ of $X$ into observation $y_{(i)}=Q^{-1}\left(p_{(i)}\right)$ of the standard normal variate $Y$, with $Q$ denoting the standard normal distribution and $Q^{-1}$ its inverse, applying discrete mapping.

For practical implementation of the methods discussed here, the commands of the freely available and widely used statistical computing language R (R Development Core Team, 2011) are provided in Appendix A (e.g. for computing the steps above).

The problems of applying the NQT arise for the reverse process, when the sampled data points in the normal space fall outside the range of historical samples (i.e. probability quantiles greater than $n /(n+1)$ or lower than $1 /(n+1))$.

In order to be able to extrapolate to extreme values, which are rarely observed in the historical samples due to the limited amount of available data, different parametric and nonparametric approaches have been tested in this paper. The problem of a sufficient amount of data is naturally very common for example in flood frequency estimation (Laio et al., 2009), downscaling of climate change scenarios for hydrological applications (Bo et al., 2007; Vrac and Naveau, 2007, 2008), and hydrology in general (Zhu, 1987; Engeland et al., 2004). This paper will specifically concentrate on the impact of small sample sizes in real-time flood forecasting using ensemble driven systems in combination with the HUP. Further discussion on unrepresentative sample sizes and the advantage of Bayesian fusion techniques can be found in Krzysztofowicz (2010).

In the next section a forecast example of the European Flood Alert System (EFAS) is shown in order to demonstrate the problem of the back-transformation and its impact on the predictive uncertainty. Then several different solutions are given and some advantages and disadvantages outlined. Finally some concluding remarks and practical advice is given. However only a very limited number of flood events have been observed during the operational run of the postprocessor and therefore the evaluation of the forecast quality is rather subjective and has to be analysed in more detail. For example the predictive QQ plot (Laio and Tamea, 2007; Thyer et al., 2009; Bogner and Pappenberger, 2011) could be used to assess whether the time-varying predictive distribution of stream-flow is consistent with the observations or similar quantile assessment methods described in Coccia and Todini (2011) could be applied once more forecast data are available.

\section{Example forecast}

The EFAS (Thielen et al., 2009; Bartholmes et al., 2009) produces daily stream-flow forecasts and includes postprocessing through data assimilation and error correction at selected stream-flow gauging stations.

Since 2010 ensembles of stream-flow forecasts issued daily are corrected and predictive uncertainties are estimated at some stations, for which historical time-series of simulations and observations are available as well as observations in real-time (see for details Bogner and Pappenberger, 2011).

In Table 1 characteristic values from two selected stations are given, Bohumin (Odra river, CZ) and Hofkirchen (Danube, DE), which will be analysed in more detail. For the calibration of the post-processor, time series of daily observed and simulated stream-flow data are necessary, which can be quite different in length and occurrences of floods at the various stations investigated. For example the data at station Bohumin comprises six years only with a maximum observed discharge of less than $600 \mathrm{~m}^{3} \mathrm{~s}^{-1}$, which corresponds roughly to a flood event with a return period between two and five years, whereas at Hofkirchen eight years of data are available including some severe flood events with twenty to 


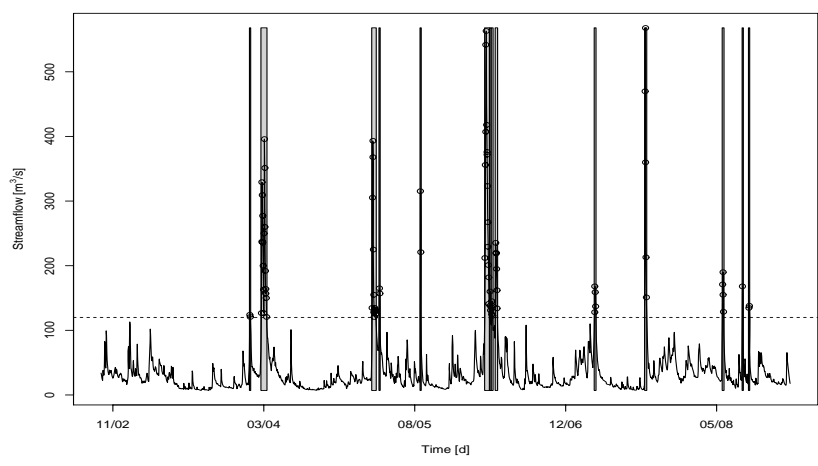

(a) Bohumin

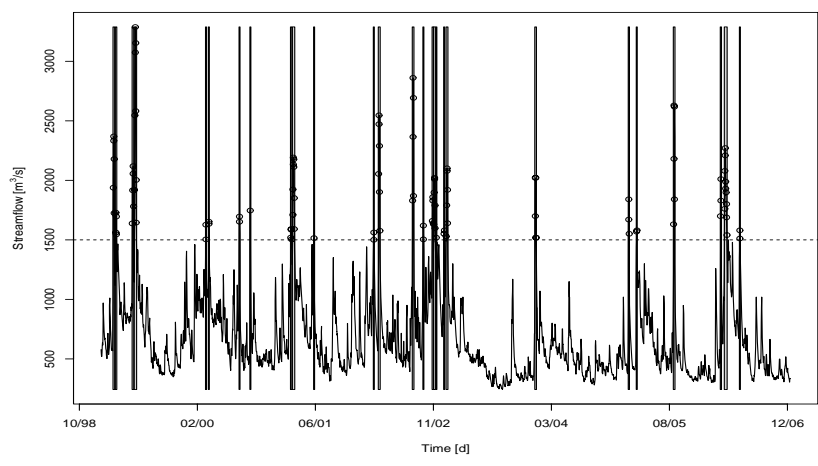

(b) Hofkirchen

Fig. 1. Observed stream-flow and number of clusters exceeding a threshold at station (a) Bohumin (Odra, CZ) and (b) Hofkirchen (Danube, DE) with 6, resp. 8 yr of daily observations.

fifty years return periods (see Fig. 1). In 2010 during the testing period for the EFAS post-processor, the forecast discharge at Bohumin far exceeded the maximum of the historical data sample, and this led to the initiation of this study. Consequently we present the case of Bohumin here. At station Hofkirchen the effect of sample size on the extrapolation methods will be analysed in more detail by comparing the results of the total available and a split sample (divided into two halves).

The post-processor runs operationally twice a day and includes the minimization of the error between the most recent past observed and simulated discharge values and the correction of the deterministic ten days ahead forecasts and the corresponding forecasts derived from two different Ensemble Prediction Systems (EPS). The NQT is applied prior to the post-processing to all available stream-flow data (measured, simulated and predicted). After the normalization step the differences between observed and simulated stream-flow values are minimized applying the Vector AutoRegressive model with eXogenous input (VARX) to the transformed time series of wavelet coefficients (i.e. fitting the VARX to the wavelet transformed series of the observations and simulations simultaneously covering a range of scales). The results of the error corrected predictions are transformed back from normal space into the real world and the predictive uncertainty is estimated (see Bogner and Pappenberger, 2011 for details). In Fig. 2 the final output of the post-processor is shown with forecast initiation at time-step zero (dashed vertical line). The past eight days $(-8, \cdots,-1)$ are included to demonstrate the performance of the error correction showing the corrected one step ahead predictions, the observations and the prediction uncertainties estimated by the HUP (Krzysztofowicz and Kelly, 2000). From lead-time one onwards $(1, \cdots, 10)$ the error corrected forecasts are shown including two stream-flow forecasts based on deterministic weather forecast systems (DWD, ECMWF-det.) and two ensemble prediction systems (51 members EPS from ECMWF and 16 members COSMO-LEPS). The resulting total predictive uncertainty integrating the model input uncertainty (i.e the weather forecast uncertainty) and the hydrological uncertainty is calculated for the different lead-times and is shown as shaded areas. Additionally two thresholds are indicated, the MQ value (lower horizontal line) representing the mean daily average discharge and the MHQ (upper horizontal line) representing the daily mean annual maximum discharge.

In Fig. 2a and d the problem of back-transforming data values exceeding the maximum of the historical sample used for calibration is demonstrated. For the observations corresponding to the one step ahead predictions, the NQT has been applied and the back-transformed measurements can show discrepancies to the real observations, because of the upper limit (observed maximum) in the historical data set. That is the reason why the observed values in Fig. 2a at timestep zero (forecast initiation) and leadtime one do not match the real observed data, but correspond to the much lower historical observed maximum. This limitation clearly indicates the necessity for including methods for extrapolation. It should be noted that this problem of extrapolation will only occur in the case of applying empirical NQT's, that means transforming empirical CDF's, and could be circumvented by fitting a theoretical CDF, e.g. the Weibull distribution, to the historical data sets (Krzysztofowicz, 1999). However the fitting of theoretical distribution functions is quite difficult under non stationary conditions and for data sets showing long-range dependencies, what is typically the case for hydrological time-series. That is the reason why the error correction method developed by Bogner and Pappenberger (2011) works with wavelet coefficients. The transformation of the time-series into the wavelet domain result in stationary and almost decorrelated variables, for which the intrinsic assumptions of the HUP are fulfilled. Nonetheless for different regions probably different distribution functions will be optimal, which would make a supervised fitting and detailed verification at each single station (i.e. stream-flow pixel in spatially distributed models) nearly impossible, especially 


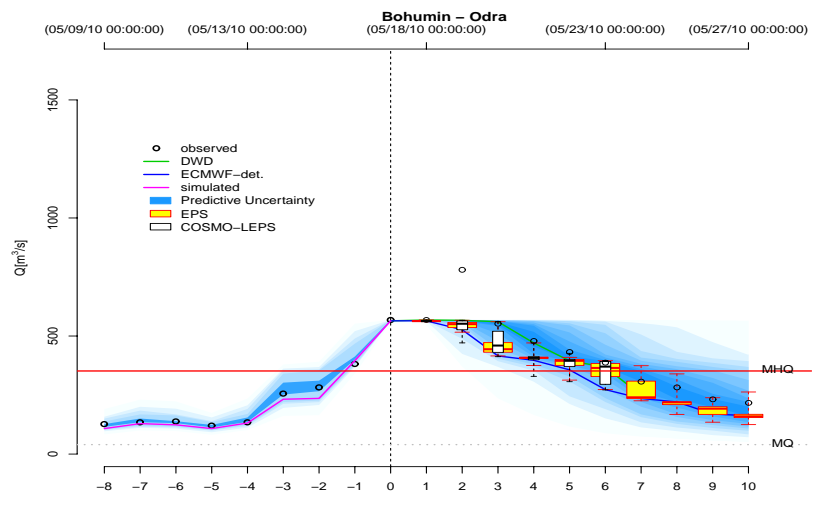

(a) Bohumin

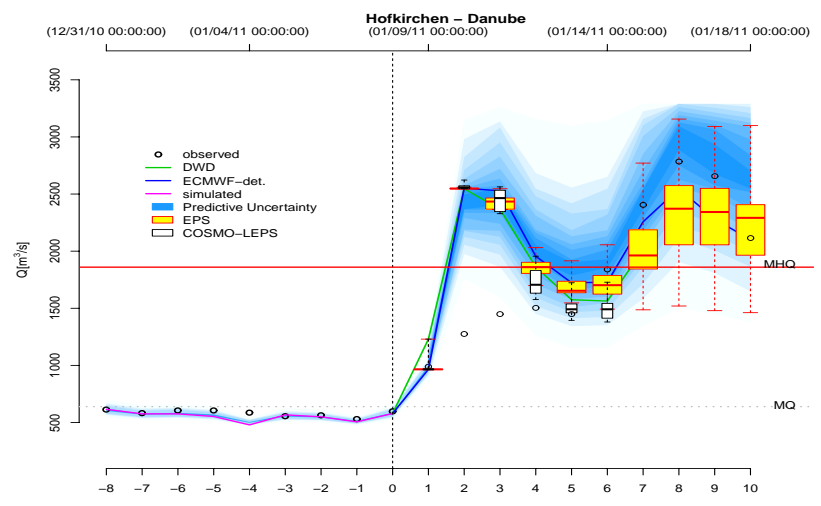

(c) Hofkirchen 4 years (first half)

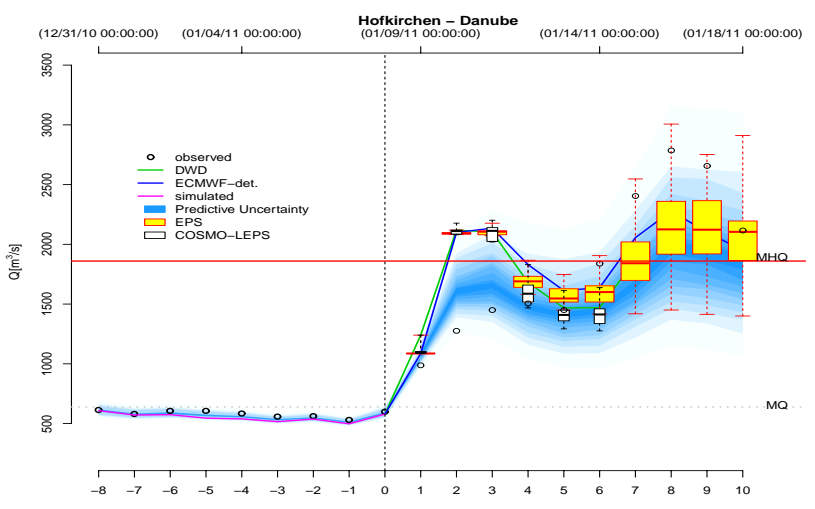

(b) Hofkirchen 8 years

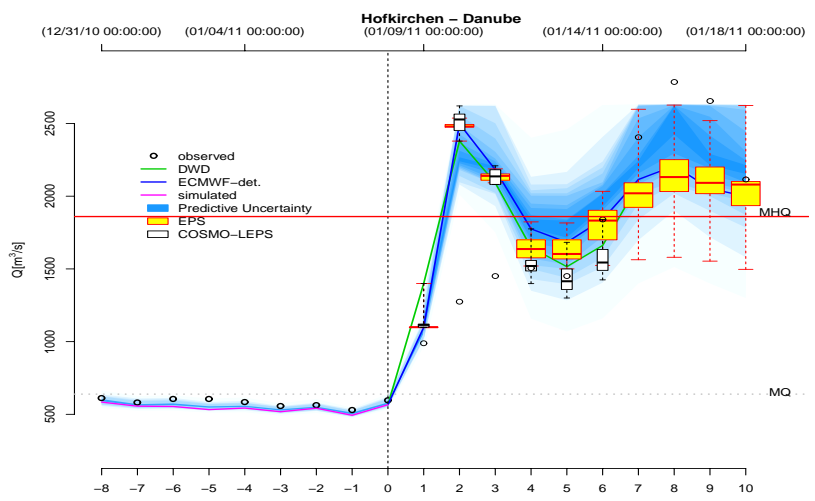

(d) Hofkirchen 4 years (second half)

Fig. 2. Corrected forecast of a flood event without extrapolation in May 2010 at station Bohumin (a), and in January 2011 at Hofkirchen taking eight years of observation (b), eliminating the first half of the data set (c), eliminating the second half of the data set (d). At station Bohumin (a) and in the second reduced sample at station Hofkirchen (d) the upper limit of applicability is shown, when the maximum of the historical sample is exceeded during the forecast period.

for continental scale forecast systems such as EFAS which runs on a $5 \mathrm{~km}$ grid over the whole of Europe.

\section{Extrapolation methods}

In this paper we concentrate on two approaches which represent a large class of possibilities and allow us to evaluate them in a flood forecasting specific setting:

1. The first method is based on extreme value theory and tries to estimate future possible extreme stream-flow values by fitting a theoretical distribution to the upper (and lower) tail of the sample. The resulting extreme values are combined with the historical sample in order to find an optimal transform function. There are multiple approaches which could be used for extrapolation based on extreme value theory, for example: normal and log-normal distributions and 3-parameter lognormal; Log-Pearson Type III; Extreme Value type I, II, or III; Generalized Extreme Value (GEV); Logistic and
General logistic; Goodrich/Weibull distribution; Exponential distribution; or Generalized Pareto Distribution (GPD) - to name a few. More mathematical and statistical details concerning extreme value theory can be found for example in Coles (2001) and Finkenstädt and Rootzén (2004).

2. In contrast to this approach the second is a nonparametric regression method called Generalized Additive Model (GAM, Hastie and Tibshirani, 1986), where the regression (i.e. transformation) function is estimated directly without specifying its parametric form explicitly. GAMs are Generalized Linear Models (GLMs, McCullagh and Nelder, 1989) in which the linear predictor is specified partly in terms of a sum of smooth functions of covariates and have been introduced for modelling non-linear relationships (Hastie and Tibshirani, 1990; Wood, 2000, 2006). Generalized additive mixed models have been proposed for over-dispersed and correlated data, which arise frequently in hydrology 
(Lin and Zhang, 1999) and that gave the reason also for choosing the GAM in this study. Many more different non-linear (regression) models exist, such as neural networks, non-linear prediction methods (e.g. Laio et al., 2003) and kernel based support vector machines (e.g. Yu et al., 2006), but their application is beyond the scope of this technical note. In Fig. 3 some non-linear relationships between the standardized normal observations and the observations are shown demonstrating the appropriateness of GAMs in fitting this non-linear transformation function.

\subsection{Extreme values}

The application of extreme value theory in hydrology has a long tradition and an associated large literature. Fisher and Tippett (1928) started to work on the asymptotic theory of extremes, whereas in Gnedenko and Kolmogorov (1949/1954) the theory for independent identically distributed random variables was completed. Fitting methods of extreme value type distributions to reliability data are outlined thoroughly in the famous work of Gumbel (1958). The Gumbel distribution is frequently applied, which is a double exponential distribution representing the limiting distribution for Gaussian data. Recently the GEV for annual maxima series (e.g. Ailliot et al., 2011) and the GPD for maxima exceeding thresholds have found the most attraction in environmental extreme value analysis (e.g. MacKay et al., 2011; Moloney and Davidsen, 2011; Mazas and Hamm, 2011).

For the practical implementation in $\mathrm{R}$ several packages are available, like "ismev", "evir" and "POT" (Ribatet, 2006), which has been used in this study. In the Peaks Over Threshold (POT) model the limiting distribution of normalised excesses over a threshold converges to the GPD, as the threshold approaches the endpoint (Pickands, 1975; Davison and Smith, 1990).

Although Hosking and Wallis (1987) recommended the method of probability weighted moments for small sample sizes, the following two-step approach has been applied here. At first the GPD parameters were estimated by minimizing the Kolmogorov-Smirnov (KS) goodness-of-fit statistics, which were taken in step two as initial values for optimizing the maximum likelihood function by the use of the NelderMead method (Nelder and Mead, 1965) resulting in stable parameter estimates and good agreements between the fitted and the empirical maxima (Fig. 4). The disadvantage of the POT model is the somewhat subjective choice of a threshold $u$ and the necessity to de-cluster the possible serial correlated time-series by defining some criterion for making the observed events independent (i.e. defining the minimum timespan between two consecutive events not exceeding the threshold, see also Bogner et al., 2012, for more details). In the example of $\mathrm{R}$ commands given in Appendix A the observed data series $x$ is de-clustered first by defining a

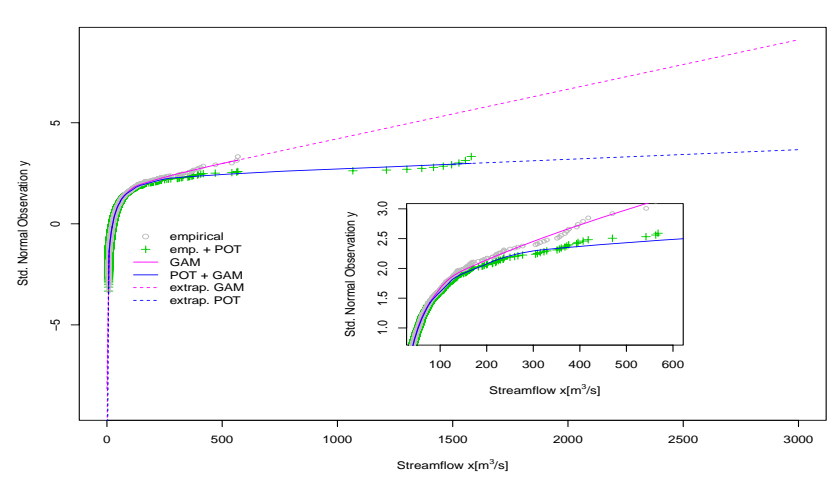

(a) Bohumin

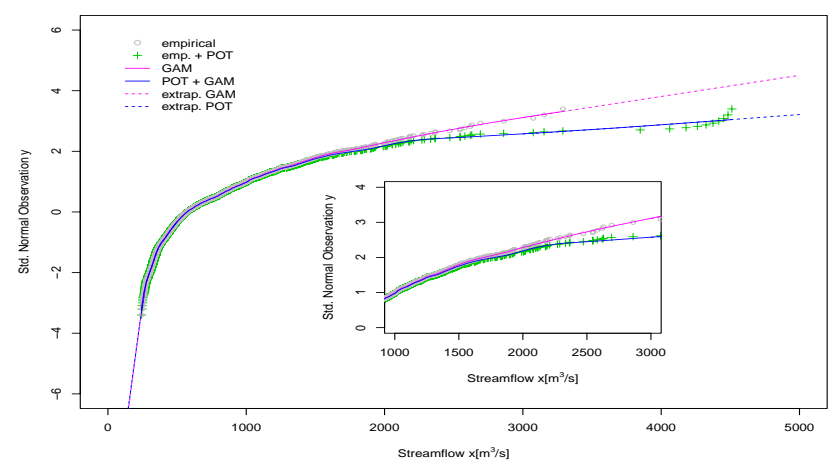

(b) Hofkirchen 8 years

Fig. 3. Normalization of stream-flow values $X$ at station Bohumin (a) and at Hofkirchen $-8 \mathrm{yr}$ (b). The range of observations with and without including extreme values (labelled as "emp. + POT", resp. "empirical") has been extrapolated applying GAMs.

timespan of eight days to ensure independence of events (see also Fig. 1).

\subsection{Nonparametric regression}

One remarkable property of the GAM is it's flexibility, permitting the data to influence the shape of the smooth functions used for exploring the relationship between the transformed variable (response) and the observation (explanatory variable). In allowing user defined penalization in the fitting by the use of natural splines it is possible to extrapolate to extreme values, without creating some unrealistic artefacts, like arbitrary and impossible swings, resulting for example from curve fitting methods based on higher degree polynomials. Natural cubic splines, which are constructed of piecewise third-order polynomials with continuity conditions expressed until second derivatives (Hastie and Tibshirani, 1990), are constrained to be linear outside the range of the data, and therefore provide a useful tool for extrapolation purposes. Note that requiring linearity outside the range of the data imposes additional smoothness constraints inside 


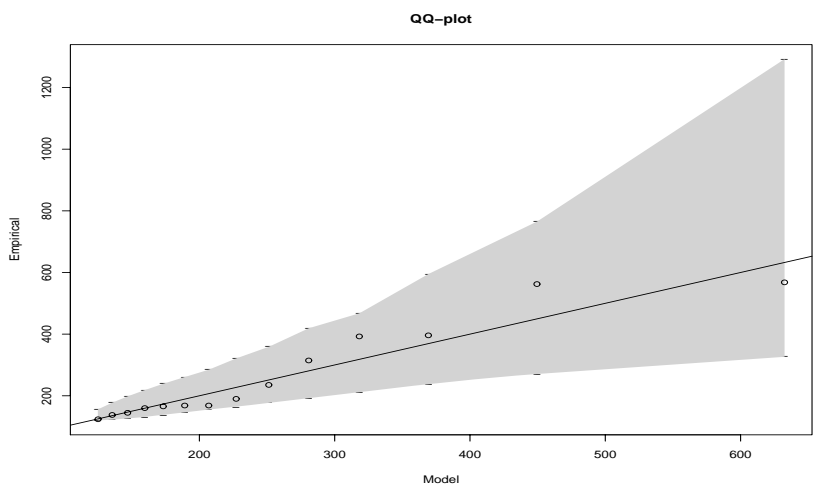

(a) Bohumin

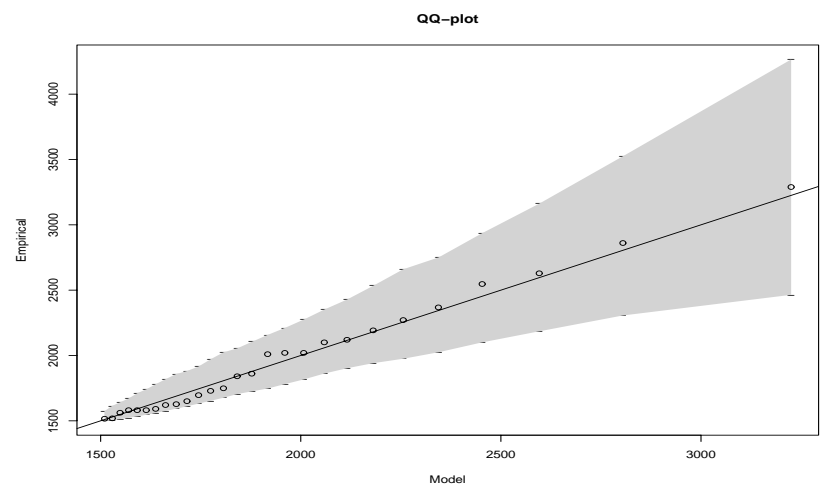

(b) Hofkirchen 8 years

Fig. 4. Resulting QQ plot for the fitted extreme value distribution (POT Model) versus empirical extreme values including the $95 \%$ confidence interval in gray for Bohumin (a) and Hofkirchen 8 yr (b).

the range. Within the R package "mgcv" (Wood, 2006) recent developments of GAMs have been implemented and the corresponding $\mathrm{R}$ function is given in Appendix $\mathrm{A}$.

\section{Results}

In order to evaluate the dependence of the NQT on the sample size and its effect on the predictive uncertainty based on the meta Gaussian distribution, two different cases have been analysed with respect to (a) extreme values, (b) GAM based and (c) combined extreme values plus GAM based extrapolation. The two different cases are:

1. Small sample size (six years) at station Bohumin including no severe flood events.

2. Sample at station Hofkirchen.

- Covering eight years (i.e. the complete data set available, including severe events).
- After elimination of the first half of the data set (reduced time series excluding most of the severe events, which are concentrated in the first half of the observed data set).

- After elimination of the second half of the data set (most of the severe events remain included).

\subsection{Station Bohumin - $6 \mathrm{yr}$}

The available data set at station Bohumin has been very limited and the sample includes almost no severe events. Therefore the flood event in May 2010 is well suited for testing extrapolation methods in view of the NQT and deriving rules for application. In the historical data set the observed maximum was about $600 \mathrm{~m}^{3} \mathrm{~s}^{-1}$, whereas in May 2010 the stream-flow values reached a maximum of approximately $1000 \mathrm{~m}^{3} \mathrm{~s}^{-1}$, which corresponds to a flood event with a return period of approximately twenty years (information provided by the Czech Hydrometeorological Institute, CHMI). Starting from the situation shown in Fig. 2a the historical data set has first been extended including extreme values derived from the POT model. The non-linear transformation function is shown in Fig. 3a with the extended sample including extreme values shown as green dots. Applying to this "statistically" extended data set the NQT, the post-processor will result in the corrected forecast including the predictive uncertainty as shown in Fig. 5a. Comparing the predictive uncertainty estimated by the GAM based inter- and extrapolation of the non-linear back-transformation function of the NQT (Fig. 5b) with the enormous range of uncertainty in the forecast of the extreme value extended sample the drawback of this method becomes quite obvious. Although this huge uncertainty range corresponds quite well with the large uncertainty range of the extreme values estimated by the POT method (Fig. 4a), from the decision maker's point of view the worth of the outcome of such an uncertainty band becomes questionable. Such enormous uncertainty ranges will probably not be accepted from the end-users of flood forecast system even when they would reflect the true uncertainty of the forecast. Obviously the definition of huge uncertainty ranges is quite subjective and it will be necessary to evaluate the different methods in a more objective way taking into consideration more flood events and more stations. From the preliminary comparison of the three different approaches in Fig. 5 one can see that the GAM based uncertainty range is by far smaller than the ranges derived from methods including extreme values, which could lead to an unrealistic impression of sharpness of the forecast system, i.e. not explaining all possible sources of uncertainty intrinsic to the flood forecasts. The last method of extrapolation is the combination of (a) extending the sample by extreme values estimated by the POT model and (b) GAM fit and extrapolation of this extended sample. In Fig. 5c the result of this method labelled "GAM + POT" is shown. The advantage of this method is the smooth transition between observed and extrapolated 


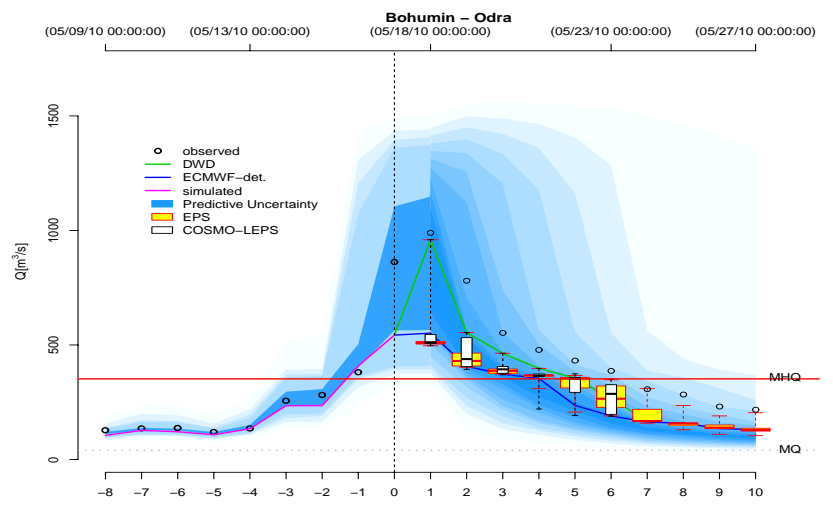

(a) Bohumin - including extreme values

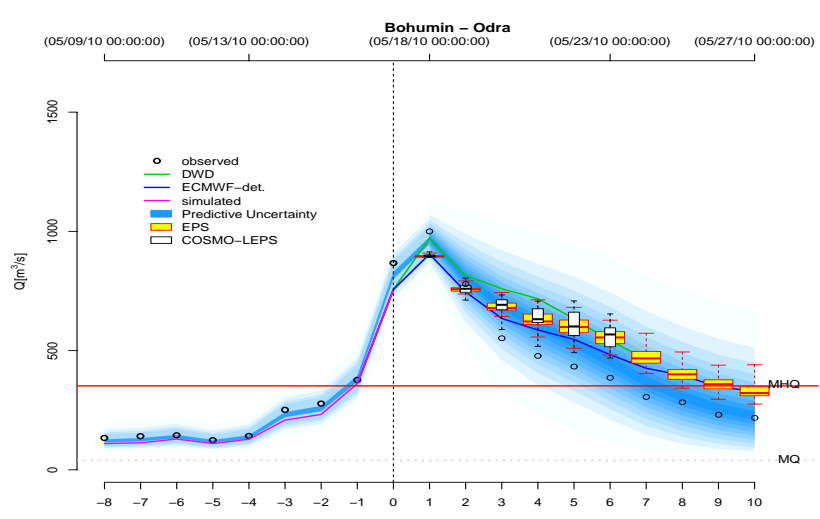

(b) Bohumin - GAM

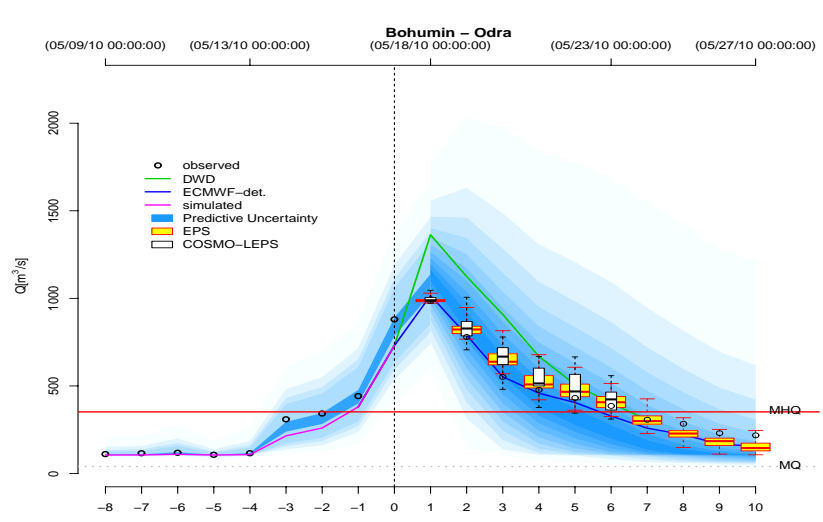

(c) Bohumin - GAM + POT

Fig. 5. Resulting forecast at station Bohumin applying three different extrapolation methods: including extreme values based on the POT method (a), fitting the GAM to the historical measurements and linear extrapolation (b), combining the POT and the GAM (c).

extreme values as opposed to the result of extending the sample size with extreme values only without applying GAM, which could result in sharp jumps in uncertainty as soon as the estimated values exceed the historical observed maximum value. In Fig. 5a this drastic discontinuity occurs at time-step zero (at forecast initiation), where the innermost quantile range (corresponding to the uncertainty range between 0.45 and 0.55 and shown by the darkest shaded area) covers a range of more than $500 \mathrm{~m}^{3} \mathrm{~s}^{-1}$. Furthermore it can be seen how a slight difference in the stream-flow value in the normal space can cause a big difference in the real world after back-transformation, like the differences in the DWD deterministic forecast (based on weather forecasts provided by the German Weather Service) ranging from $1000 \mathrm{~m}^{3} \mathrm{~s}^{-1}$ in Fig. $5 \mathrm{a}$ and b to $1300 \mathrm{~m}^{3} \mathrm{~s}^{-1}$ in Fig. 5c. However in Fig. 5c it can be seen that the inner quantile ranges $(0.35-0.65)$ cover all the observations in the forecast period (from leadtime 1 to 10) and the predicted median follows quite well the observations, whereas the first two methods result in under or over predictions from a leadtime of three days onwards.

\subsection{Station Hofkirchen}

The historical data sample at station Hofkirchen comprises eight years and includes several quite severe flood events such as those in May 1999, spring 2002 and August 2002, which correspond to flood events with return periods between 10 and $20 \mathrm{yr}$ (information provided by the Bavarian Environmental Agency). Given this representative data set several tests have been conducted analysing the NQT performance and its dependence on sample size and on the extrapolation method. Since the majority of the flood events occurred in the first half of the sample (between 1998 and 2003) the NQT and the post-processor have been applied on the total sample and on the split (halved) sample (Fig. 2bd). Taking the total data set of eight years for calibrating the post-processor results in a forecast with a predictive uncertainty covering almost all the observations, although the first peak of this double-peaked event is overestimated and the second one, the more severe peak, is underestimated (Fig. 2b). Nonetheless the observed values of the second peak fall within the upper quantile range $(0.05-0.95)$ at a leadtime of eight days, which is quite good for a medium range forecast. Fitting the post-processor to the first half of the sample size (Fig. 2c) leads to an increased uncertainty because of the greater variability of the sample. In comparison to this the calibration based on the second half sample results in to a forecast with smaller uncertainty bands because of the smaller variance of the sample, demonstrating the importance of sample size and explaining the upper limit of applicability of the NQT, when the observations exceed the historical sample (Fig. 2d).

In the case of the eight years data set, the artificial extension and/or the application of the GAM approach only resulted in modest changes in the forecast and consequently these results are not shown here. However taking only the first half of the sample with several severe events results in an extreme value distribution producing too heavy tails and therefore heavily overshooting predictive uncertainty ranges in the forecast example (Fig. 6a). The inclusion of 


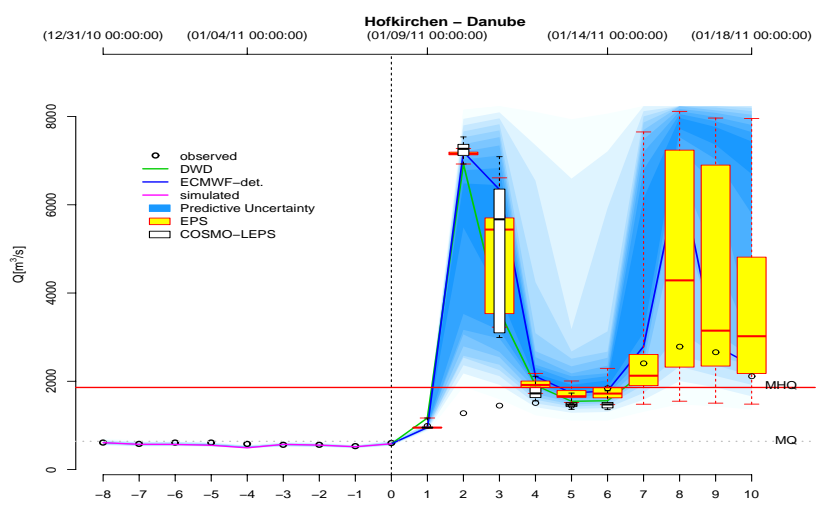

(a) Hofkirchen 4 years (first half, POT)

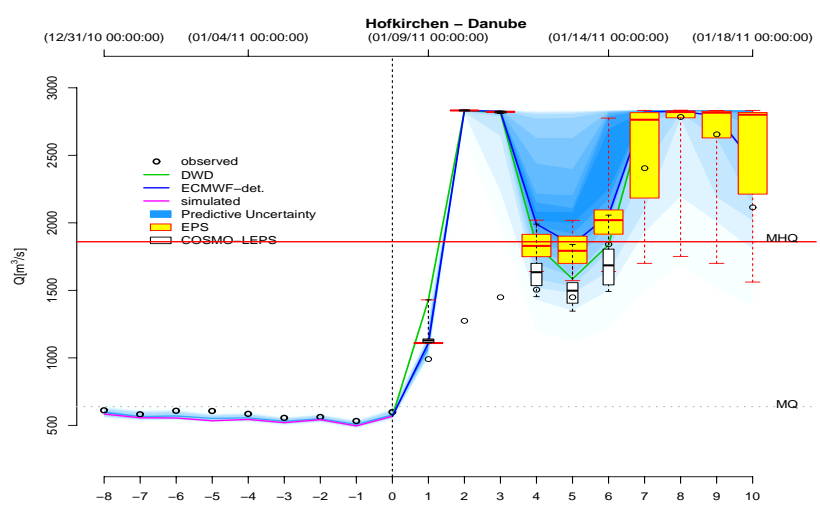

(b) Hofkirchen 4 years (second half, POT)

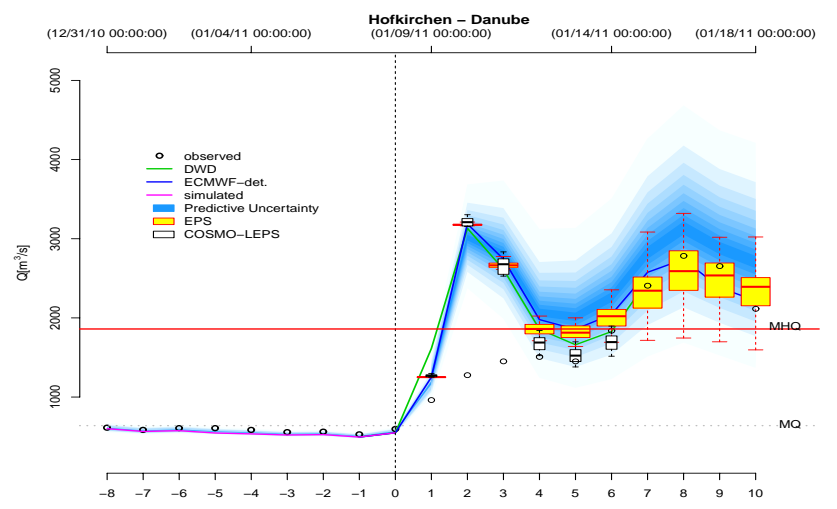

(c) Hofkirchen 4 years (second half, GAM + POT)

Fig. 6. Corrected forecast at station Hofkirchen including extreme values for extrapolation based on (a) four years of the first half, including several severe events and (b) four years of the second half (less severe events) and (c) four years of the second half sample combining the GAM and the POT model for extrapolation.

extreme values fitted to the second half sample results in back-transformed forecast values which substantially exceed the observations because of the discontinuity between the historical data set and the estimated extreme values. Such discontinuities will result in unrealistic, abrupt changes as can be seen in Fig. 6b at leadtime three and can be circumvented by the application of the GAM in combination with the POT model, which will result in smooth forecasts (Fig. 6c).

\section{Conclusions}

In this study of the applicability of the NQT in flood forecasting systems different problems arising from the small sample sizes are discussed. The chosen forecast examples at two different stream-flow gauging stations and for two different flood events demonstrate the problems of extending the historical data set by extreme values resulting from the fitted POT model. Because of the discontinuity between the observed historical sample and the estimated extreme values, sharp and unrealistic rises (or falls) in the hydrograph can occur after transforming the forecast from the Gaussian to the "real world" space. The analysed GAM for approximating the non-linear (back)transformation function could be an alternative, but the problem of possibly unrealistic small predictive uncertainty ranges has to be investigated in more detail with longer time-series and at different stations. However for these very limited data sets analysed the suggested way would be the combination of the extension of the small samples by extreme values and the inter- and extrapolation of this prolonged data set by the GAM, which results in smooth forecast hydrographs and not too optimistic under-dispersive predictive uncertainty ranges.

\section{Appendix A}

\section{Listing of $\mathbf{R}$ commands}

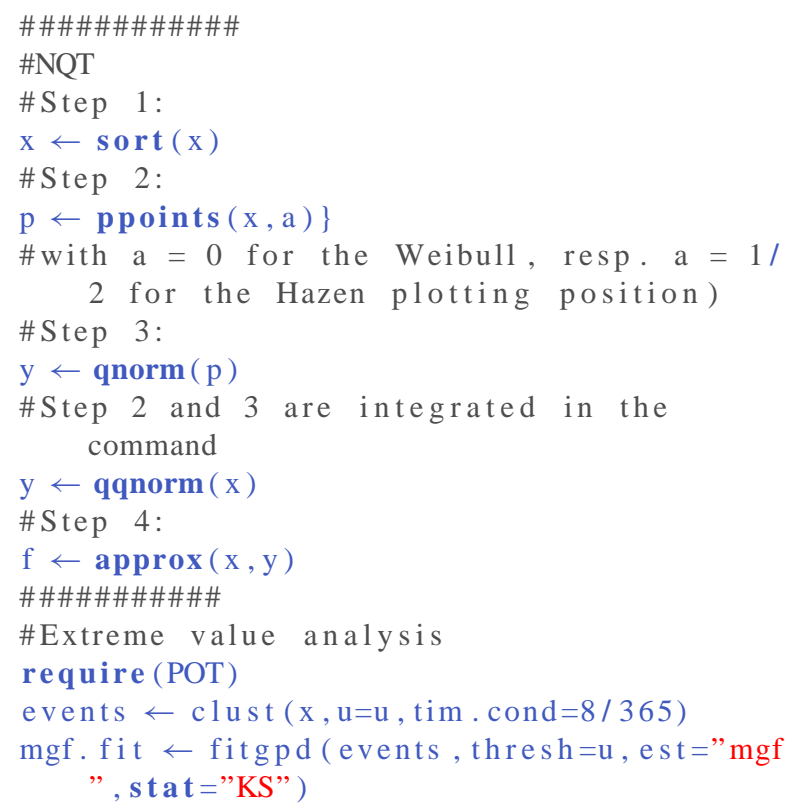




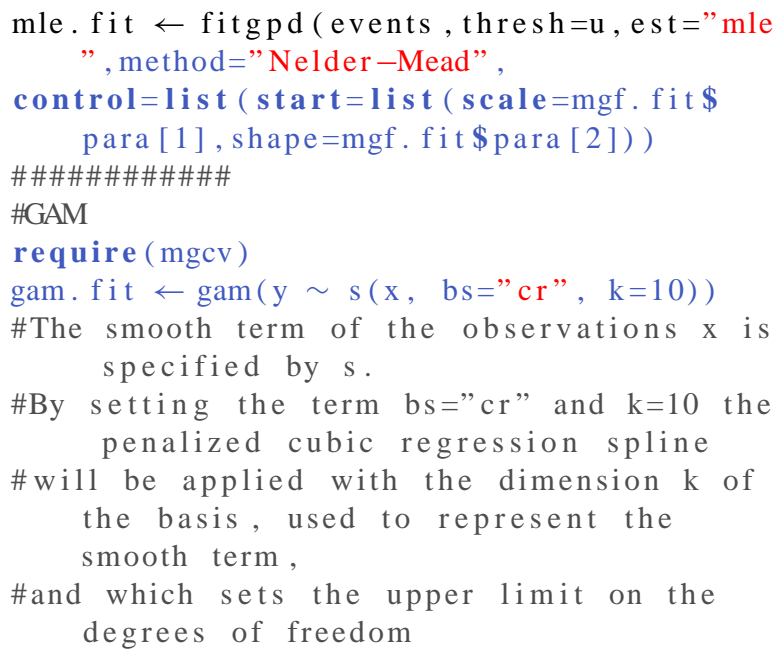

Acknowledgements. The authors wish to thank the Deutsche Wetterdienst, the European Centre for Medium-Range Weather Forecasts, the Bavarian Environment Agency, the Czech Hydrometeorological Institute (CHMI) and the JRC's Institute for Protection and Security of the Citizen (IPSC) for data and information. The work was also supported by the UK Natural Environment Research Council (grant NE/I005366/1). Florian Pappenberger is partially funded by GLOWASIS (Global Water Scarcity Information Service) project (EC, FP7) and DEWFORA (Drought Early Warning Forecasts, EC, FP7). Finally the authors gratefully acknowledge the support of all staff of the JRC's Institute for Environment and Sustainability (IES) - Land Management and Natural Hazards Unit - FLOODS Action.

Edited by: C. de Michele

\section{References}

Ailliot, P., Thompson, C., and Thomson, P.: Mixed methods for fitting the GEV distribution, Water Resour. Res., 47, W0551, doi:10.1029/2010WR009417, 2011.

Bartholmes, J. C., Thielen, J., Ramos, M. H., and Gentilini, S.: The european flood alert system EFAS - Part 2: Statistical skill assessment of probabilistic and deterministic operational forecasts, Hydrol. Earth Syst. Sci., 13, 141-153, doi:10.5194/hess-13-1412009, 2009.

Bo, J., Terray, L., Habets, F., and Martin, E.: Statistical and dynamical downscaling of the Seine basin climate for hydrometeorological studies, Int. J. Climatol., 1655, 1643-1655, 2007.

Bogner, K. and Pappenberger, F.: Multiscale error analysis, correction, and predictive uncertainty estimation in a flood forecasting system, Water Resour. Res., 47, W07524, doi:10.1029/2010WR009137, 2011.

Bogner, K., Cloke, H., Pappenberger, F., De Roo, A., and Thielen, J.: Improving the evaluation of hydrological multi-model forecast performance in the Upper Danube Catchment, Int. J. River Basin Manage., 10, 1-12, doi:10.1080/15715124.2011.625359, 2012.
Coccia, G. and Todini, E.: Recent developments in predictive uncertainty assessment based on the model conditional processor approach, Hydrol. Earth Syst. Sci., 15, 3253-3274, doi:10.5194/hess-15-3253-2011, 2011.

Coles, S.: An introduction to statistical modeling of extreme values, Springer series in statistics, Springer, 2001.

Davison, A. C. and Smith, R. L.: Models for Exceedances over High Thresholds, J. Roy. Stat. Soc. B, 52, 393-442, 1990.

Deutsch, C. and Journel, A.: GSLIB: Geostatistical Software Library and User's Guide, 2nd Edn., Oxford University Press, USA, 1998.

Engeland, K., Hisdal, H., and Frigessi, A.: Practical Extreme Value Modelling of Hydrological Floods and Droughts: A Case Study, Extremes, 7, 5-30, doi:10.1007/s10687-004-4727-5, 2004.

Finkenstädt, B. and Rootzén, H.: Extreme values in finance, telecommunications, and the environment, Monographs on statistics and applied probability, Chapman \& Hall/CRC, 2004.

Fisher, R. A. and Tippett, L. H. C.: Limiting forms of the frequency distributions of the largest or smallest member of a sample, Proc. Cambridge Philos. Soc., 24, 180-190, 1928.

Gnedenko, B. V. and Kolmogorov, A. N.: Limit Distributions for Sums of Independent Random Variables, Gosudarstv. Izdat Tehn.-Teor. Lit., MoscowLeningrad, 1949 in Russian, English transl.: Addison-Wesley, Cambridge, Mass., 1954.

Goovaerts, P.: Geostatistics for Natural Resources Evaluation, Applied Geostatistics Series, Oxford University Press, USA, 1997.

Gumbel, E. J.: Statistics of extremes, 2nd Edn., Columbia University Press, New York, 1958.

Hastie, T. and Tibshirani, R.: Generalized Additive Models (with discussion), Stat. Sci., 1, 297-318, 1986.

Hastie, T. and Tibshirani, R.: Generalized Additive Models, Chapman and Hall, London, 1990.

Hosking, J. and Wallis, J.: Parameter and quantile estimation for the generalized Pareto distribution, Technometrics, 29, 339-349, 1987.

Kelly, K. and Krzysztofowicz, R.: A bivariate meta-Gaussian density for use in hydrology, Stoch. Hydrol. Hydraul., 11, 17-31, 1997.

Krzysztofowicz, R.: Transformation and normalization of variates with specified distributions, J. Hydrol., 197, 286-292, 1997.

Krzysztofowicz, R.: Bayesian theory of probabilistic forecasting via deterministic hydrologic model, Water Resour. Res., 35, 2739-2750, 1999.

Krzysztofowicz, R.: Decision Criteria, Data Fusion, and Prediction Calibration: A Bayesian Approach, Hydrolog. Sci. J., 55, 10331050, 2010.

Krzysztofowicz, R. and Herr, H. D.: Hydrologic uncertainty processor for probabilistic river stage forecasting: precipitationdependent model, J. Hydrol., 249, 46-68, 2001.

Krzysztofowicz, R. and Kelly, K.: Hydrologic uncertainty processor for probabilistic river stage forecasting, Water Resour. Res., 36, 3265-3277, 2000.

Krzysztofowicz, R. and Maranzano, C. J.: Hydrologic uncertainty processor for probabilistic stage transition forecasting, J. Hydrol., 293, 57-73, 2004.

Laio, F. and Tamea, S.: Verification tools for probabilistic forecasts of continuous hydrological variables, Hydrol. Earth Syst. Sci., 11, 1267-1277, doi:10.5194/hess-11-1267-2007, 2007. 
Laio, F., Porporato, A., Revelli, R., and Ridolfi, L.: A comparison of nonlinear flood forecasting methods, Water Resour. Res., 39, 1129, doi:10.1029/2002WR001551, 2003.

Laio, F., Di Baldassarre, G., and Montanari, A.: Model selection techniques for the frequency analysis of hydrological extremes, Water Resour. Res., 45, W07416, doi:10.1029/2007WR006666, 2009.

Lin, X. and Zhang, D.: Inference in generalized additive mixed modelsby using smoothing splines, J. Roy. Stat. Soc. B, 61, 381400, doi:10.1111/1467-9868.00183, 1999.

MacKay, E., Challenor, P., and Bahaj, A.: A comparison of estimators for the generalised Pareto distribution, Ocean Eng., 38, 1338-1346, 2011.

Mazas, F. and Hamm, L.: A multi-distribution approach to POT methods for determining extreme wave heights, Coast. Eng., 58, 385-394, 2011.

McCullagh, P. and Nelder, J. A.: Generalized linear models, 2nd Edn., Chapman \& Hall, London, 1989.

Moloney, N. and Davidsen, J.: Extreme bursts in the solar wind, Geophys. Res. Lett., 38, L14111, doi:10.1029/2011GL048245, 2011.

Montanari, A.: Deseasonalisation of hydrological time series through the normal quantile transform, J. Hydrol., 313, 274-282, 2005.

Montanari, A. and Brath, A.: A stochastic approach for assessing the uncertainty of rainfall-runoff simulations, Water Resour. Res., 40, W01106, doi:10.1029/2003WR002540, 2004.

Montanari, A. and Grossi, G.: Estimating the uncertainty of hydrological forecasts: A statistical approach, Water Resources Research, 44, W00B08, doi:10.1029/2008WR006897, 2008.

Moran, P.: Simulation and Evaluation of Complex Water Systems Operations, Water Resour. Res., 6, 1737-1742, 1970.

Nelder, J. A. and Mead, R.: A Simplex Method for Function Minimization, Comput. J., 7, 308-313, 1965.

Pickands, J.: Statistical inference using extreme order statistics, Ann. Stat., 3, 119-131, 1975.

R Development Core Team: R: A Language and Environment for Statistical Computing, ISBN 3-900051-07-0, R Foundation for Statistical Computing, http://www.R-project.org/, Vienna, Austria, 2011.

Reggiani, P., Renner, M., Weerts, A., and van Gelder, P.: Uncertainty assessment via Bayesian revision of ensemble streamflow predictions in the operational river Rhine forecasting system, Water Resour. Res., 45, W02428, doi:10.1029/2007WR006758, 2009.
Ribatet, M. A.: A User's Guide to the POT Package (Version 1.0), http://cran.r-project.org/ (last access: March 2012), 2006.

Seo, D.-J., Herr, H. D., and Schaake, J. C.: A statistical postprocessor for accounting of hydrologic uncertainty in short-range ensemble streamflow prediction, Hydrol. Earth Syst. Sci. Discuss., 3, 1987-2035, doi:10.5194/hessd-3-1987-2006, 2006.

Thielen, J., Bartholmes, J., Ramos, M.-H., and de Roo, A.: The European Flood Alert System - Part 1: Concept and development, Hydrol. Earth Syst. Sci., 13, 125-140, doi:10.5194/hess-13-1252009, 2009.

Thyer, M., Renard, B., Kavetski, D., Kuczera, G., Franks, S., and Srikanthan, S.: Critical evaluation of parameter consistency and predictive uncertainty in hydrological modeling: A case study using Bayesian total error analysis, Water Resour. Res., 45, W00B14, doi:10.1029/2008WR006825, 2009.

Todini, E.: A model conditional processor to assess predictive uncertainty in flood forecasting, Int. J. River Basin Manage., 6, 123-137, 2008.

Van der Waerden, B. L.: Order tests for two-sample problem and their power I, Indagat. Math., 14, 453-458, 1952.

Van der Waerden, B. L.: Order tests for two-sample problem and their power II, Indagat. Math., 15, 303-310, 1953a.

Van der Waerden, B. L.: Order tests for two-sample problem and their power III, Indagat. Math., 15, 311-316, $1953 \mathrm{~b}$.

Vrac, M. and Naveau, P.: Stochastic downscaling of precipitation: From dry events to heavy rainfalls, Water Resour. Res., 43, W07402, doi:10.1029/2006WR005308, 2007.

Vrac, M. and Naveau, P.: Correction to "Stochastic downscaling of precipitation: From dry events to heavy rainfall", Water Resour. Res., 44, W05702, doi:10.1029/2008WR007083, 2008.

Weerts, A. H., Winsemius, H. C., and Verkade, J. S.: Estimation of predictive hydrological uncertainty using quantile regression: examples from the National Flood Forecasting System (England and Wales), Hydrol. Earth Syst. Sci., 15, 255-265, doi:10.5194/hess-15-255-2011, 2011.

Wood, S.: Modelling and smoothing parameter estimation with multiple quadratic penalties, J. Roy. Stat. Soc. B, 62, 413-428, 2000.

Wood, S.: Generalized Additive Models: An Introduction with R, Chapman and Hall/CRC, 2006.

Yu, P., Chen, S., and Chang, I.: Support vector regression for realtime flood stage forecasting, J. Hydrol., 328, 704-716, 2006.

Zhu, J.: A discussion on the extrapolation of hydrologic series, J. Hydrol., 96, 225-235, 1987. 